\title{
The Role of Heterogeneity and Centrality for Promoting Cooperation in Prisoner's Dilemma Games
}

\author{
Ge Xin, Li Lili \\ College of Information Science and Technology, Dalian Maritime University, Dalian, China \\ Ge_xin@dlmu.edu.cn, lilili@dlmu.edu.cn
}

Keywords: Prisoner’s dilemma games, network reciprocity, cooperation, centrality.

\begin{abstract}
In the evolutionary games based on a heterogeneous population, recent researches has shown that the degree of each player in the network plays an important role and often determines the level of cooperation. Yet, the individual influence described by centralities remain inadequate in quantifying the effect of promoting cooperation. In this paper, how the representative centralities impact the fate of cooperation on different levels of heterogeneous populations has been comprehensively investigated. Simulation results show that on the whole, centrality characteristic is efficient to facilitate cooperation in social dilemmas except the clustering, and degree is neither the sole nor the best one. Meanwhile, there is an optimal level of heterogeneity that maximizes the cooperators regardless of the influence of centralities.
\end{abstract}

\section{Introduction}

How the large-scale cooperation emerges, sustains and evolves has become a challenging subject for many different fields from biology, economic to social sciences. The evolutionary game theory ${ }^{[1,}$ ${ }^{2]}$ provides a convenient and fundamental framework for the elucidation of this domain ${ }^{[3]}$. In the well-known Prisoner's Dilemma (PD) games, two players (individuals) interact with each other through strategies of Cooperation (C) and Defection (D). They both get a reward $R$ for mutual cooperation and a punishment $P$ for mutual defection. If one player cooperates while the other defects, their game payoffs are $S$ (sucker's payoff) and $T$ (temptation), respectively. The dilemma occurs when $T>R>P>S$, because the optimum choice for both players is defection regardless of opponent's strategy. One of seminal works solve this dilemma is performed by Nowak ${ }^{[4]}$ who proposed direct and spatial reciprocity or referred as network reciprocity. Meanwhile, compelling evidence has been accumulated that a plethora of biological, social, and technological real-world networks of contacts are mostly heterogeneous ${ }^{[5,6]}$. That is to say, the roles of different nodes may be significantly impact the evolutionary dynamics in real-world structured populations. To quantitatively measure the roles of different nodes, numerous centrality metrics have been proposed, among which most studies have focused on node degree, e.g., proportion payoffs by the number of individual neighborhood, namely degree ${ }^{[7]}$. However, the networks with identical degree distribution perhaps exhibit great difference in regarding of individual importance. Then, one natural question is: 
to what extent and in what manner the centralities affect evolutionary behavior and cooperation level?

To achieve a comprehensive insight of this issue, a systematic study and simulations have been performed based on PD games, taking into account seven most representative metrics of individual centralities on prototypical model for heterogeneous structure of populations. Here, this paper is interested in the distinctive role of different centralities on promoting cooperation, especially which centralities are efficient metric identifying cooperative hubs. Centralities is incorporated via preferentially assigning strategy among populations according to individual centrality scores. This paper reveals a nontrivial role of the population structure indicated by heterogeneity and centrality in the evolution of cooperation.

\section{Methods}

\subsection{Network model}

The structured heterogeneous populations can be modeled through mapping players to nodes of heterogeneous network. The Barabasi-Albert $(B A)$ model provides the best known model leading to overall scale-free degree distributions $d(k)=k^{-\gamma}$. In this study, we adopt the single-scale version implemented by Eppstein David, etc. ${ }^{[8]}$ (here referred as $E D$ model) because it reflects the real-world connectivity particular the social relationship such as acquaintances. Besides strength of heterogeneity, another crucial difference between these two models is that $B A$ model doesn't contain node of $k=1$, leading to all the nodes have identical Coreness. While Coreness is one vital metric to measure the influence of individuals, which has attracted intensive attention and has been as a baseline in the comparison of different centralities ${ }^{[9]}$. To avoid stochastic effect, we have checked larger network size up to $N=10^{5}$ and smaller one, down to values of $N=100$ We found that populations size $N>1500$ is able to remain results unchanged thus in the present study, the simulations were performed for the setup of $k=2000$ and the average degree $<\mathrm{k}>=2-8$.

\subsection{Game model}

In the PD game, we use payoff matrix $R=2$ and $P=1$ and correspondingly $0 \leq S \leq 1$ and $2 \leq T \leq 3$. The studied region in the $T$-S plane we employed was sampled in steps of 0.1 , thus encompassing $11 \times 11=121$ parameter combinations. The evolutionary process with synchronous update procedure comprises discrete elementary steps (game round) where the whole population play simultaneously. The traditional process (referred as benchmark simulation) consists of two steps, initialization and update. Initially, a portion of individuals $\rho_{C}(0)$ (here 0 indicates the first time step) randomly chosen are assigned cooperation strategy and others defection strategy. As to strategy updating, player $x$ with strategy $s_{x}$ imitates the strategy $s_{y}$ of another player $y$, chosen randomly from the neighborhood of $x$, iff $y$ 's strategy has yielded higher payoff $p_{y}$, otherwise player $x$ maintains its original strategy. Player $x$ takes over the strategy $s_{y}$ with a probability determined by Fermi rule ${ }^{[10]}$.

$$
\mathcal{P}\left(s_{y}^{t} \rightarrow s_{x}^{t+1}\right)=\frac{1}{1+e^{-\left[\left(p_{y}^{t}-p_{x}^{t}\right) / k\right]}}
$$

Where $k=0.1$ quantifies the uncertainty related to the strategy changing process. The selected value of $k$ is a traditional and frequently employed choice that does not qualitatively affect the evolutionary outcomes. 


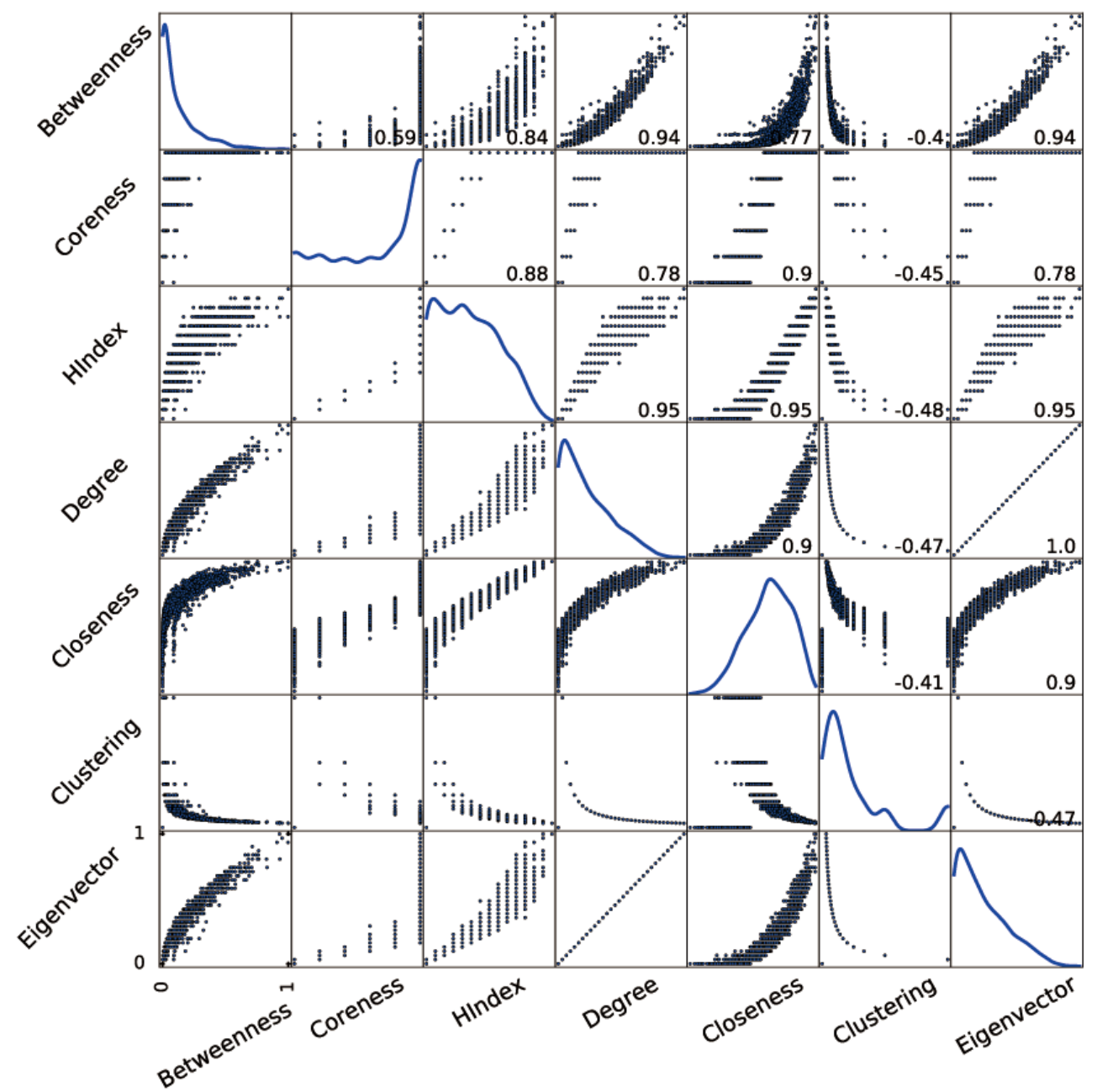

Fig.1. Correlation matrix and distributions of centralities considered in this study on network of $\langle\mathrm{k}\rangle$ $=4$. The $\varphi$ of any two centrality metrics are shown on upper triangular insets.

Since stimulating specific individuals to cooperate is a feasible and applicable approaches in the purpose of promoting cooperation. All the players are ranked according to a centrality metric then select top- $I$ ones as initial cooperators, namely $\rho_{C}(0)=I / N$. We employ the centrality metrics Degree(Deg), Hindex(Hin), Coreness(Cor), Clustering(Clu), Closeness(Clo), Eigenvector(Eig) and Betweenness(Bet) measuring influential populations. It is vital to investigate the correlation between any two centrality metrics used in the present study. As detailed in Fig.1, we computed the Pearson correlation coefficients $\varphi$ between any two centrality metrics in networks of $<k>=4$. The outcomes indicate that strong linear correlations do exist between certain centrality metrics. It is natural to expect they maybe have akin impact on evolutionary dynamic. Note that for Degree and Eigenvector $\varphi=1$, which means the same set of nodes will be got according to sorted scores of these two metrics, thus in the next section, the Eigenvector is omitted in the results.

For the population size, $N=2000$ and times well over 5000 steps warrants a correct convergence and steady outcomes, in agreement with many other works in this field, like for example in [11]. To measure the cooperation level, a quantitative measure $\mathcal{C}$ is used for the overall asymptotic cooperation in the PD game, given by averaging the $\rho_{c}(\infty)$ over the corresponding region in the $T-S$ plane. Moreover, to assure suitable accuracy and the stochastic effect all the final results are obtained via averaging 100 independent runs for each set of parameters. 


\section{Results and Discussion}

\subsection{Effect of heterogeneity}

To assess the fundamental evolution of PD game, this paper presents the result of benchmark dynamic in Figs. 2 and 3 which illustrate the impact of average degree $<\mathrm{k}>$ and initial proportion of cooperators $\rho_{C}(0)$ on the outcome of the $T-S$ space panel. It is clearly evidenced that heterogeneity drastically boosts cooperation compared with the evolution underlying random and well-mixed population that inevitably evolves to full defection. Despite the heterogeneity generally play a positive role in driving cooperation, we need scrutinize the results in terms of the correlation between heterogeneity and final evolutionary state. In the ED model, the level of heterogeneity $\gamma$ is proportional to $<\mathrm{k}>$, therefore each column of Fig.2 (e.g., panel a, d, g, j) records the final abundance of cooperators with the heterogeneity varying from lower level $(\gamma=-1.8)$ to higher level $(\gamma=-2.6)$. According to I intuition, cooperation should gradually thrive with the average degree increasing due to the consensus that interconnectivity between networks does promote cooperation by means of enhanced reciprocity, yet the influence of heterogeneity is not monotonous toward cooperation level, $<\mathrm{k}>=2$ being the exception from $<\mathrm{k}>=4,6,8$. To suppress the bias of stochastic disturbance and fluctuation, we further inspect the conditions of smaller resolution of $<\mathrm{k}>$ and the result is given in Fig.3. It shows that irrespective of initial fraction of cooperators the mean $\rho_{C}(0)$ on $T-S$ panel reaches the maximum at $\langle\mathrm{k}>=2.7$ (see panel $\mathrm{j}, \mathrm{k}, \mathrm{i}$ ). This result suggests that the cooperation level does not monotonously depend on the connection density of underlying network(e.g., the $\rho_{C}(0)=0.13,0.19,0.18,0.27,0.26$ corresponding to $<\mathrm{k}>=2.0,2.3,2.7,2.4$ when $\left.\rho_{C}(0)=0.3\right)$, in other words, existing an intermediate heterogeneity optimally sustaining cooperation. It implies that if a population structure evolves to become increasingly or decreasingly heterogeneous beyond the threshold, the population will eventually reach a structure under which cooperation becomes less viable as shown in the panel a of Fig.3. It can be predicted that the cooperators will thoroughly die out on $T-S$ panel when degree exceeds one threshold that closes to the average degree of wellmixed structure. Moreover, this result is robust to variations of $\rho_{C}(0)$, and thus indicate a high degree of universality. Previous work has focused on the mechanism of network reciprocity, while the existence of threshold lacks interpretation. Those individuals with more connections have more opportunity to participate in the majority of the interactions. In this case, they accumulate large fitness based on payoffs and determine the outcome of evolution. However, if the overall connection is too sparse (lower degree) or too dense (higher degree), it is difficult for independent formation of cooperative patterns on each individual network due to the asymmetric strategy flow.

\subsection{Effect of centrality}

In this section, this paper proceeds exploring the evolution of cooperation considering nonrandom strategy assignment, one applicable and feasible approaches to foster cooperation. The results under varying heterogeneity $<\mathrm{k}>=2,4,8,2.7$ are depicted separately in Figs.4, 6, 7 and 5, corresponding to the first, second and fourth rows of Fig.2 and the fourth row of Fig.3, respectively. Notably, we have assessed the conditions where $<\mathrm{k}>=2$ to 3 at interval 0.1 and $<\mathrm{k}>=3$ to 8 at interval 1, while only present the representative results corresponding to the benchmark result in Fig.2 and 3. 


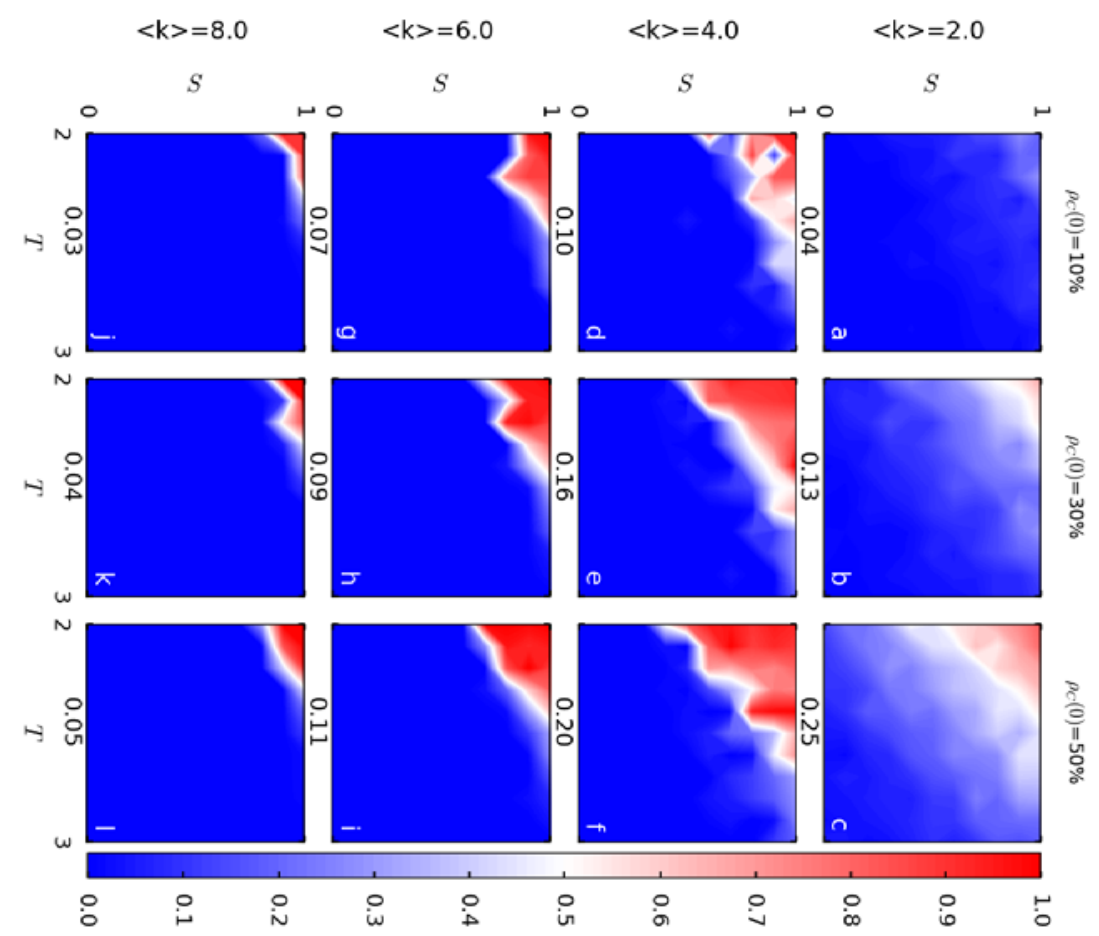

Fig.2. Asymptotic density of cooperators $\mathcal{C}$ on different heterogenous structured populations, $<\mathrm{k}>$ $=2,4,6,8$.

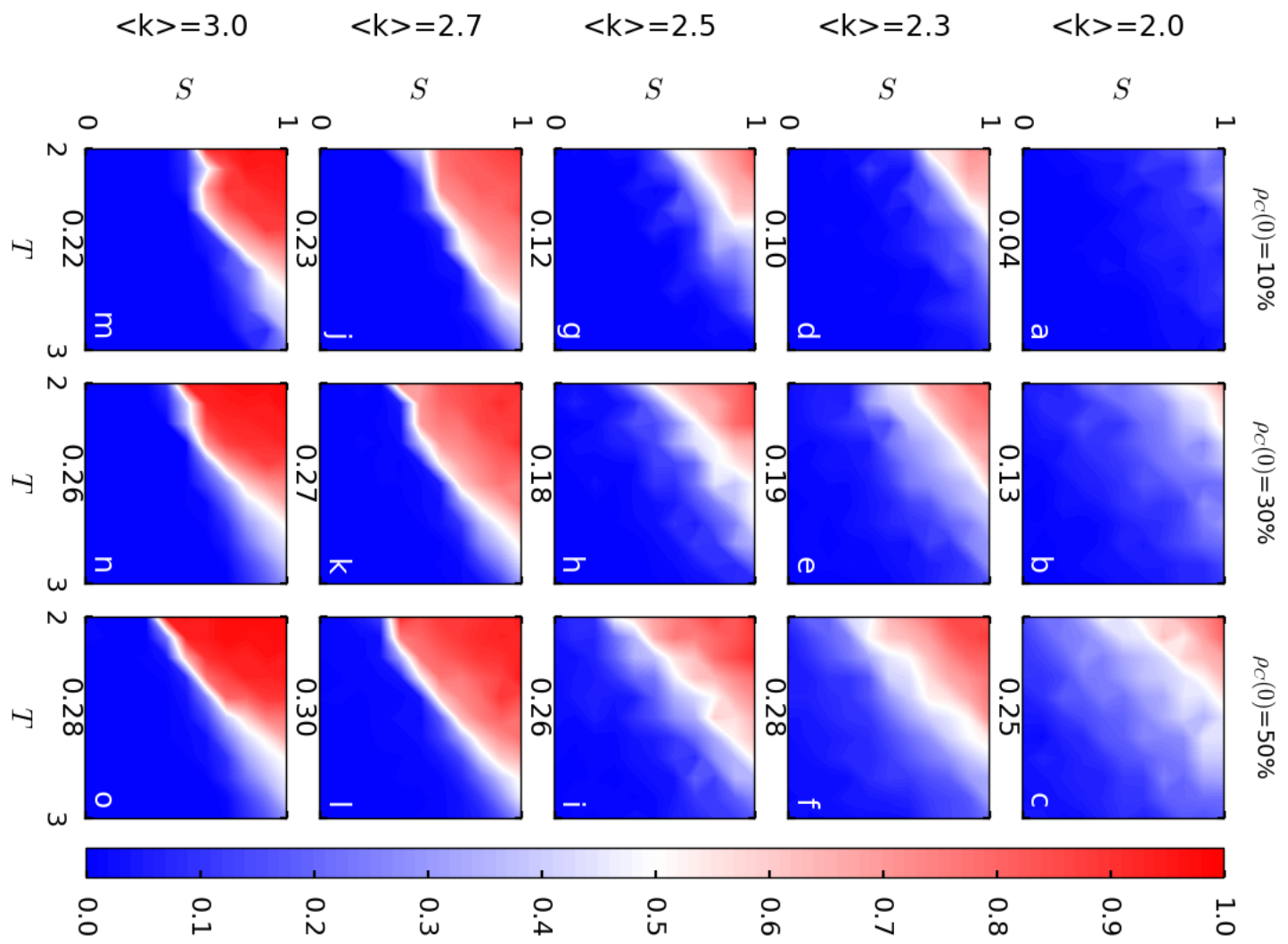

Fig.3. Asymptotic density of cooperators $\mathcal{C}$ on different heterogenous structured populations, $<\mathrm{k}>$ $=2,2.3,2.5,2.7,3$. 
HIndex Degree Coreness Clustering Closeness Betweenness

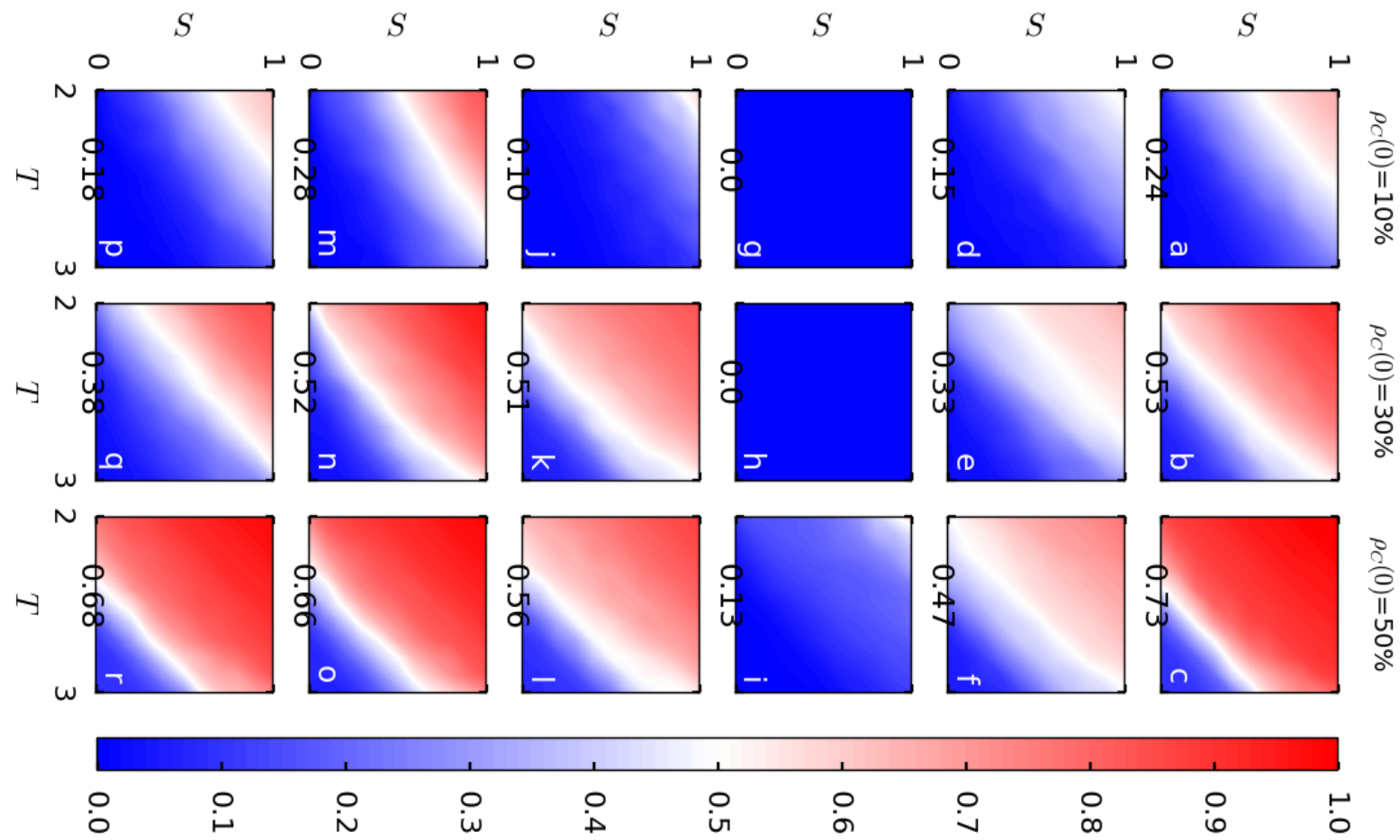

Fig.4. Asymptotic density of cooperators $\mathcal{C}$ in $<\mathrm{k}\rangle=2$, centrality preferential assignment of strategy.

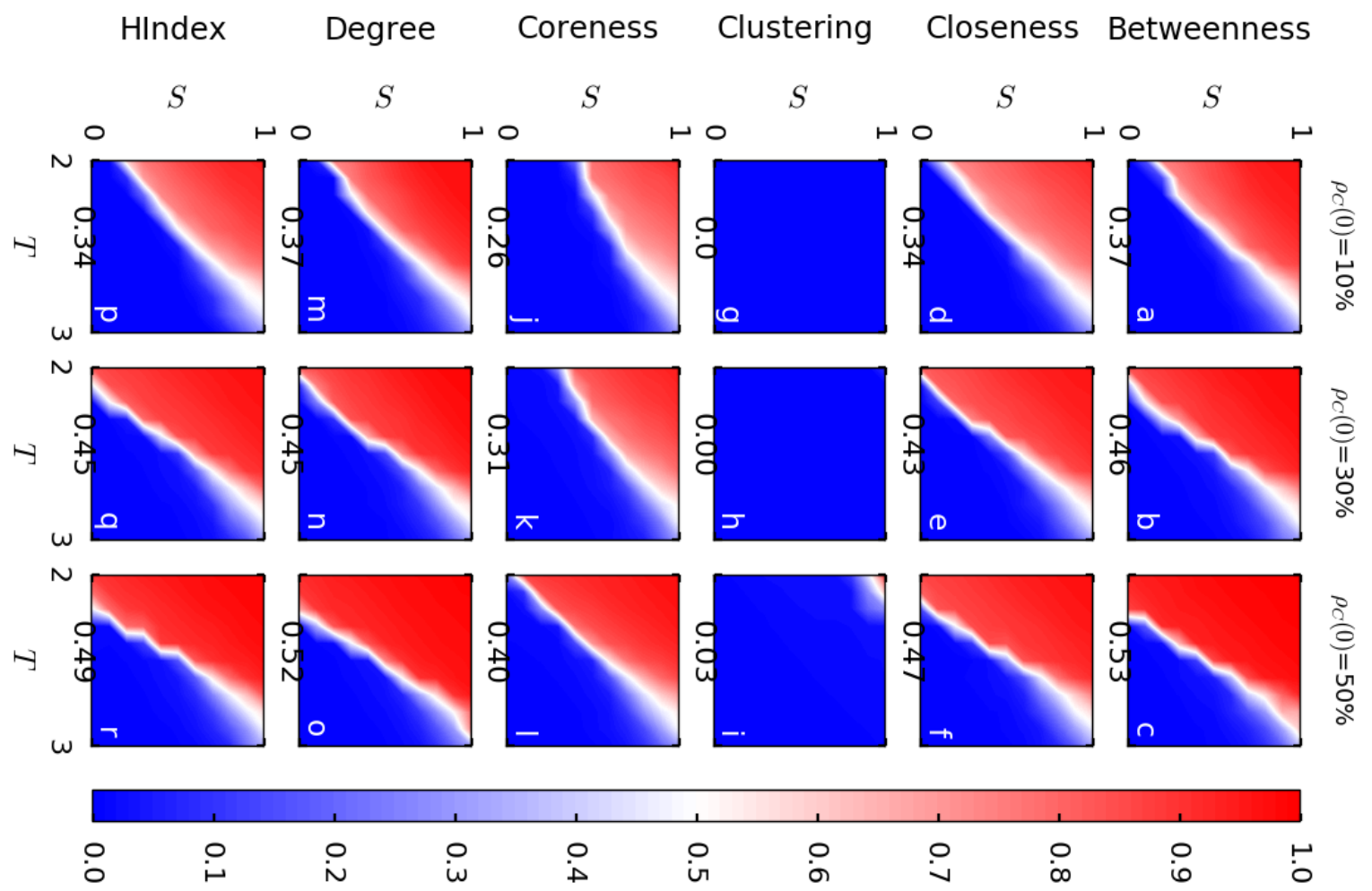

Fig.5. Asymptotic density of cooperators $\mathcal{C}$ in $\langle\mathrm{k}\rangle=2.7$, centrality preferential assignment of strategy 
In most cases, preferential initialization elevates the final level of cooperation, which happens irrespectively of the initial fraction of cooperators and, more importantly, of the $<\mathrm{k}>$. For instance between panels m, n, o in Fig. 2 and panels j, k, l in Fig.6 , the mean level of cooperation rises from $0.1,0.16$ and 0.2 to $0.18,0.22$ and 0.57 when initially designate cooperators based on Coreness metric. Aside from aforementioned result, another considerable difference between random initiation in benchmark and centrality preferential initiation is that the optimal level of heterogeneity facilitating cooperation shifts from $<\mathrm{k}>=2.7$ to $<\mathrm{k}>=2$. Accordingly, a natural question is that whether $\langle\mathrm{k}\rangle=2$ is the most optimal level amongst all available values of $\langle\mathrm{k}>$, not limited to this study. In fact, the extreme condition of minimum value $<\mathrm{k}>=0$ can be considered, where each node is isolated and has no connection to others. Due to the absence of direct links between individuals, it can be inferred that each cooperators can successfully resist the invasion of defectors and remain their initial strategy. In this case, the final outcome is completely determined by the initial fraction of cooperators. For instance, if $\rho_{C}(0)=0.5$, the $\mathrm{T}-\mathrm{S}$ space will be dominated by white color(denotes 0.5 in color bar), regardless of values of $\mathrm{T}$ and $\mathrm{S}$. Apparently, $<\mathrm{k}>=0$ is not the optimal value promoting cooperation and we can predict the optimal value is between 1 and 2. These results, consequently reveal that the optimal level of heterogeneity indeed exists not only in benchmark evolution, but also in the version of preferentially initiation, and more importantly the optimal value is not identical.

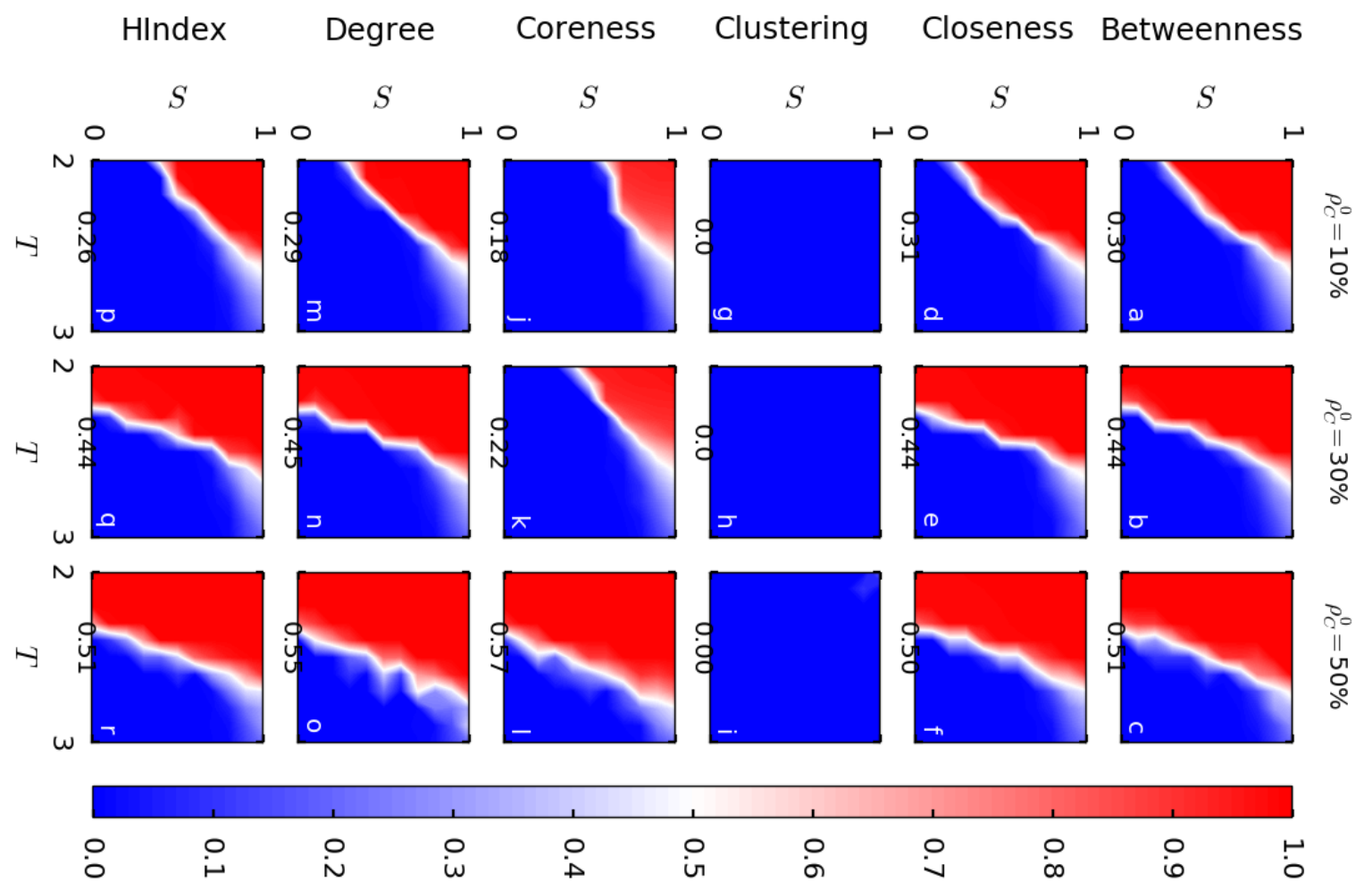

Fig.6. Asymptotic density of cooperators $\mathcal{C}$ in $<\mathrm{k}>=4$, centrality preferential assignment of strategy. 


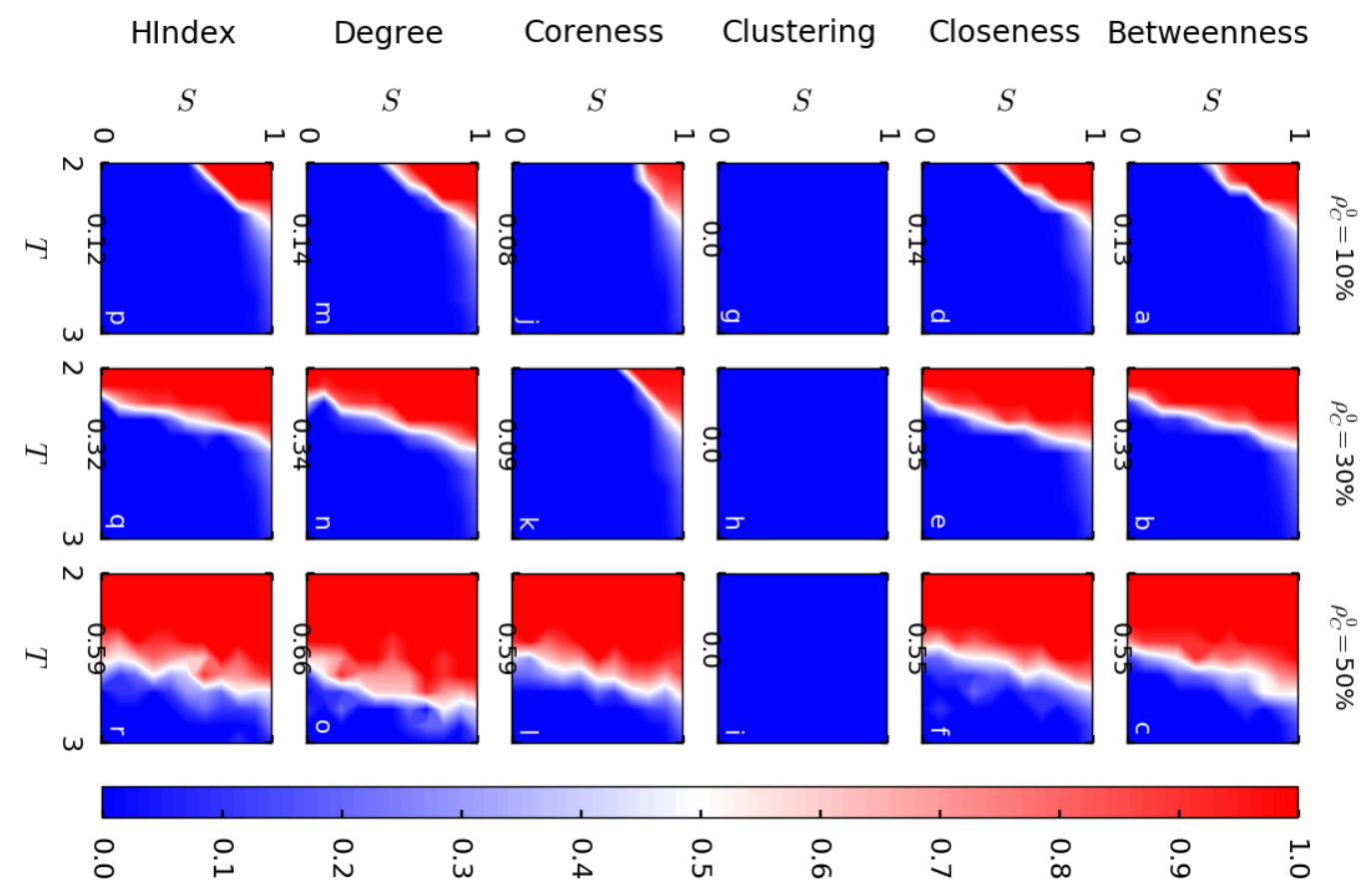

Fig.7. Asymptotic density of cooperators $\mathcal{C}$ in $<\mathrm{k}>=8$, centrality preferential assignment of strategy.

To systematically compare the impact of different centrality metric, these centralities according to $\mathrm{C}$ and dependency to $\rho_{C}(0)$ are ranked.

$$
\begin{cases}C_{\text {Deg }}>C_{\text {Bet }}>C_{\text {Hin }}>C_{\text {Clo }}>C_{\text {Cor }}>C_{C l u} & \rho_{C}(0)=0.1 \\ C_{\text {Bet }}>C_{\text {Deg }}>C_{\text {Cor }}>C_{\text {Hin }}>C_{C l o}>C_{C l u} & \rho_{C}(0)=0.3 \\ C_{\text {Bet }}>C_{\text {Hin }}>C_{\text {Deg }}>C_{\text {Cor }}>C_{C l o}>C_{C l u} & \rho_{C}(0)=0.5\end{cases}
$$

Basically, the centralities can be roughly classified into three classes according to relative effectiveness of promoting cooperation. It can be observed that the Betweenness, Degree and Hindex as the first class noticeably and stably enhance the resilience of cooperation compared with other centralities, particularly in dense and stronger heterogeneous population. The result for $<\mathrm{k}>=2.7$ is reported in Fig.5. As plots clearly show, the overall level of cooperation in this case considerably outperform that of other degrees as well as in the benchmark results. Similarly, centrality Degree, Betweenness and Hindex still play the role of better promoters and Closeness, Coreness, Cluster play worse ones, albeit the discrepancy weakens. Comparing the first three metric, we can see that Hindex is almost identical to Degree, even better in certain conditions (see Fig.4o r); And Betweenness is the most outstanding indicator in the case of $<\mathrm{k}\rangle=2$ and $<\mathrm{k}\rangle=2.7$ (see a, b, c in both Fig.4 and Fig.5). In the second class, despite Closeness and Coreness also foster cooperation, their influence is not as intense as the centralities in the first class, occasionally illustrating weak effect (see Fig.4d, e, $\mathrm{j}$ and Fig.6j, k). Lastly, the third class consisting of only Cluster always lead to the full-defection outcome, plays the opposite role, even worse than random selection of initial cooperators. This implies that Cluster thoroughly lost influence in the angle of helping cooperators.

Unlike in the benchmark cases depicted in Figs.2 and 3, the relative advantages of centrality cannot hold stabilization under the fluctuation of initial fraction of cooperators $\rho_{C}(0)$, e.g., as shown in Fig.4, in the case of $\langle\mathrm{k}\rangle=2$, Degree yields the best outcome if $\rho_{C}(0)=0.1$ (panel $\mathrm{m}$ ), whereas Betweenness becomes the optimal one if $\rho_{C}(0)=0.5$ (panel c). This phenomenon means again that the influence of initial fraction of cooperators cannot be negligible, especially as decreasing $\rho_{C}(0)$, 
the gap of $\mathrm{C}$ between different centralities extends. As illustrated, these results confirm the hypothesis that the evolutionary outcome is driven by the initial state of the most influential individuals in the population. This result reveal the limitation that a centrality which is optimal for one condition in the evolutionary game is often sub-optimal for a different condition.

\section{Conclusion}

This paper has systematically studied the evolution of cooperation on structured population where the heterogeneity can be continuously adjusted through controlling model parameter degree. Besides optimal heterogeneity, we find significant effect in promoting cooperation by deliberately initializing cooperators via ranking population, while different centralities are not able to measure evolutionary influence in general conditions, someone even counter-intuitive, e.g., the Clustering index plays a negative role in cooperative evolution. On one hand, Hindex, Coreness and Degree centralities are better indicators to locate important individuals as initial cooperators because of their prominent effect and low computational complexity. On the other hand, Degree is not the only, and in certain conditions not the most effective centrality facilitating cooperation. In this study, the centralities is directly applied to find the initial set of cooperators, which could be inefficient since their influences may be largely overlapped. Therefore, comprehensively identify a set of influential nodes emerges as a significant cooperators may be an alternative approach.

\section{Acknowledgment}

This paper is supported by Natural Science Foundation of Liaoning Province (No.20170540097) and Fundamental Research Funds for the Central Universities (No.3132018127).

\section{References}

[1] Smith, J.M. Evolution and the Theory of Games; Cambridge university press, 1982.

[2] Nowak, M.A. Evolutionary Dynamics: Exploring the Equations of Life. 2006.

[3] Trivers, R.L. The evolution of reciprocal altruism. Quarterly review of biology 1971, pp. 35-57.

[4] Nowak, M.A. Five rules for the evolution of cooperation. science 2006, 314, 1560-1563.

[5] Amaral, L.A.N.; Scala, A.; Barthelemy, M.; Stanley, H.E. Classes of small-world networks. Proceedings of the national academy of sciences 2000, 97, 11149-11152.

[6] Dorogovtsev, S.N.; Mendes, J.F. Evolution of networks: From biological nets to the Internet and WWW; OUP Oxford, 2013.

[7] Santos, F.C.; Santos, M.D.; Pacheco, J.M. Social diversity promotes the emergence of cooperation in public goods games. Nature 2008, 454, 213-216.

[8] Eppstein, D.; Wang, J. A steady state model for graph power laws. IN 2ND INTERNATIONAL WORKSHOP ON WEB DYNAMICS. Citeseer, 2002.

[9] Liu, Y.; Tang, M.; Zhou, T.; Do, Y. Core-like groups result in invalidation of identifying super-spreader by $k$-shell decomposition. Scientific reports 2015, 5.

[10] Szabó, G.; T"oke, C. Evolutionary prisoner's dilemma game on a square lattice. Physical Review E 1998, 58, 69.

[11] Wang, Z.; Wang, L.; Perc, M. Degree mixing in multilayer networks impedes the evolution of cooperation.

Physical Review E 2014, 89, 0 